\title{
ON NONUNIQUENESS FOR CALDERÓN'S INVERSE PROBLEM
}

\author{
Allan Greenleaf, Matti Lassas, and Gunther Uhlmann \\ Abstract. We construct anisotropic conductivities with the same Dirichlet-to- \\ Neumann map as a homogeneous isotropic conductivity. These conductivities are \\ singular close to a surface inside the body.
}

\section{Introduction}

An anisotropic conductivity on a domain $\Omega \subset \mathbf{R}^{n}$ is defined by a symmetric, positive semi-definite matrix-valued function, $\sigma=\left(\sigma^{i j}(x)\right)$. In the absence of sources or sinks, an electrical potential $u$ satisfies

$$
\begin{aligned}
(\nabla \cdot \sigma \nabla) u=\partial_{j} \sigma^{j k}(x) \partial_{k} u & =0 \text { in } \Omega, \\
\left.u\right|_{\partial \Omega} & =f,
\end{aligned}
$$

where $f$ is the prescribed voltage on the boundary. Above and hereafter we use the Einstein summation convention where there is no danger of confusion. The resulting voltage-to-current (or Dirichlet-to-Neumann) map is then defined by

$$
\Lambda_{\sigma}(f)=\left.B u\right|_{\partial \Omega},
$$

where

$$
B u=\nu_{j} \sigma^{j k} \partial_{k} u,
$$

$u$ is the solution of $(1)$ and $\nu=\left(\nu_{1}, \ldots, \nu_{n}\right)$ is the unit normal vector of $\partial \Omega$.

Applying the divergence theorem, we have

$$
Q_{\sigma}(f):=\int_{\Omega} \sigma^{j k}(x) \frac{\partial u}{\partial x^{j}} \frac{\partial u}{\partial x^{k}} d x=\int_{\partial \Omega} \Lambda_{\sigma}(f) f d S
$$

where $u$ solves (1) and $d S$ denotes surface measure on $\partial \Omega . Q_{\sigma}(f)$ represents the power needed to maintain the potential $f$ on $\partial \Omega$. By (4), knowing $Q_{\sigma}$ is equivalent with knowing $\Lambda_{\sigma}$. If $F: \Omega \rightarrow \Omega, \quad F=\left(F^{1}, \ldots, F^{n}\right)$, is a diffeomorphism with $\left.F\right|_{\partial \Omega}=$ Identity, then by making the change of variables $y=F(x)$ and setting $u=v \circ F^{-1}$ in the first integral in (4), we obtain

$$
\Lambda_{F_{*} \sigma}=\Lambda_{\sigma}
$$

Received March 6, 2003.

The first and third authors are partially supported by the NSF, the second author by the Academy of Finland, and the third author by a John Simon Guggenheim Fellowship. 
where

$$
\left(F_{*} \sigma\right)^{j k}(y)=\left.\frac{1}{\operatorname{det}\left[\frac{\partial F^{j}}{\partial x^{k}}(x)\right]} \sum_{p, q=1}^{n} \frac{\partial F^{j}}{\partial x^{p}}(x) \frac{\partial F^{k}}{\partial x^{q}}(x) \sigma^{p q}(x)\right|_{x=F^{-1}(y)}
$$

is the push-forward of the conductivity $\sigma$ by $F$. Thus, there is a large (infinitedimensional) class of conductivities which give rise to the same electrical measurements at the boundary. The uniqueness question we wish to address, first proposed by Calderón [C], is whether two conductivities with the same Dirichletto-Neumann map must be such pushforwards of each other. By a direct construction, we will show that the answer is no. Let $D \subset \subset \Omega$ be a smooth subdomain. We will construct in $\Omega$ a conductivity $\widehat{\sigma}$ for which the boundary measurements coincide with those made for the homogeneous conductivity $\gamma=1$ in $\Omega$. We note that the conductivity is singular in the sense that some components of the conductivity tensor go to zero, i.e., correspond to perfectly insulating directions and some components can go to infinity, i.e., correspond to perfectly conducting directions, as one approaches $\partial D$. The physical meaning of these counterexamples is evident: In medical imaging, e.g., in Electrical Impedance Tomography (EIT), certain anisotropic structures can form barriers which, including their interior, appear in measurements to be a homogeneous medium.

To construct these counterexamples, we need to consider a variant of (1), which comes from the Laplace-Beltrami operator on a compact Riemannian manifold with boundary. Let us assume now that $(M, g)$ is an $n$-dimensional Riemannian manifold with smooth boundary $\partial M$. The metric $g$ is assumed to be symmetric and positive definite. The invariant object analogous to the conductivity equation (1) is the Laplace-Beltrami operator, which is given by

$$
\Delta_{g} u=G^{-1 / 2} \partial_{j}\left(G^{1 / 2} g^{j k} \partial_{k} u\right)
$$

where $G=\operatorname{det}\left(g_{j k}\right),\left[g_{j k}\right]=\left[g^{j k}\right]^{-1}$. The Dirichlet-to-Neumann map is defined by solving the Dirichlet problem

$$
\begin{aligned}
& \Delta_{g} u=0 \quad \text { in } \quad M, \\
& \left.u\right|_{\partial M}=f .
\end{aligned}
$$

The operator analogous to $\Lambda_{\sigma}$ is then

$$
\Lambda_{g}(f)=\left.G^{1 / 2} \nu_{j} g^{j k} \frac{\partial u}{\partial x^{k}}\right|_{\partial M},
$$

with $\nu=\left(\nu_{1}, \ldots, \nu_{n}\right)$ the outward unit normal to $\partial M$. In dimension three or higher, the conductivity matrix and the Riemannian metric are related by

$$
\sigma^{j k}=\operatorname{det}(g)^{1 / 2} g^{j k}, \quad \text { or } \quad g^{j k}=\operatorname{det}(\sigma)^{2 /(n-2)} \sigma^{j k} .
$$

Moreover, $\Lambda_{g}=\Lambda_{\sigma}$, and $\Lambda_{\psi^{*} g}=\Lambda_{g}$, where $\psi^{*} g$ denotes the pullback of the metric $g$ by a diffeomorphism of $M$ fixing $\partial M$ [LeU]. 
In dimension two, (9) is not valid; in this case, the conductivity equation can be reformulated as

$$
\begin{aligned}
& \operatorname{Div}_{g}\left(\beta \operatorname{Grad}_{g} u\right)=0 \quad \text { in } \quad M, \\
& \left.u\right|_{\partial M}=f
\end{aligned}
$$

where $\beta$ is the scalar function $\beta=|\operatorname{det} \sigma|^{1 / 2}, g=\left(g_{j k}\right)$ is equal to $\left(\sigma_{j k}\right)$, and $\operatorname{Div}_{g}$ and $\operatorname{Grad}_{g}$ are the divergence and gradient operators with respect to the Riemannian metric $g$. Thus we see that, in two dimensions, Laplace-Beltrami operators correspond only to those conductivity equations for which $\operatorname{det}(\sigma)=1$.

For domains in two dimensions, Sylvester[Sy] showed, using isothermal coordinates, that one can reduce the anisotropic problem to the isotropic one; combining this with the isotropic result of Nachman[Na], one obtains

Theorem 1. If $\sigma$ and $\widetilde{\sigma}$ are two $C^{3}$ anisotropic conductivities in $\Omega \subset \mathbf{R}^{2}$ for which $\Lambda_{\sigma}=\Lambda_{\sigma}$, then there is a diffeomorphism $F: \Omega \rightarrow \Omega,\left.F\right|_{\partial \Omega}=I d$ such that $\tilde{\sigma}=F_{*} \sigma$.

In dimensions three and higher, the following result is known (see [LU], [LTU], and $[\mathrm{LeU}])$ :

Theorem 2. If $n \geq 3$ and $(M, \partial M)$ is a $C^{\omega}$ manifold with connected, $C^{\omega}$ boundary, and $g, \widetilde{g}$ are $C^{\omega}$ metrics on $M$ such that $\Lambda_{g}=\Lambda_{g}$, then there exists a $C^{\omega}$ diffeomorphism $F: M \rightarrow M$ such that $\left.F\right|_{\partial D}=I d$.

\section{Counterexamples}

Returning now to domains $\Omega \subset \subset \mathbf{R}^{n}, n \geq 3$, let $D \subset \subset \Omega$ be an open subset with smooth boundary and $g=g_{i j}$ be a metric on $\Omega$. Let $y \in D$ be such that there is a diffeomorphism $F: \Omega \backslash\{y\} \rightarrow \Omega \backslash \bar{D}$, and let $\widetilde{g}=F_{*} g$ on $\Omega \backslash \bar{D}$. To obtain a conductivity on all of $\Omega$, first extend $\widetilde{g}$ to a bounded metric inside $D$ and denote this new metric on $\Omega$ by $\widehat{g}$. We make this continuation so that the conductivity jumps on $\partial D$ and that $\widehat{g} \geq c>0$ is smooth inside $D$, but is otherwise arbitrary. Let $\widehat{\sigma}$ be the conductivity corresponding to $\widehat{g}$ by (9) in $\Omega$. We say that $v$ is a solution of the conductivity equation if

$$
\begin{aligned}
& \nabla \cdot \widehat{\sigma} \nabla v(x)=0 \text { in the sense of distributions in } \Omega, \\
& \left.v\right|_{\partial \Omega}=f_{0}, \\
& v \in L^{\infty}(\Omega)
\end{aligned}
$$

where $f_{0} \in H^{\frac{1}{2}}(\partial \Omega)$. That the equation in the sense of distributions means that $v \in H^{1}(\Omega)$ and $\sigma \nabla v \in H(\Omega ; \nabla \cdot)=\left\{w \in L^{2}\left(\Omega ; \mathbf{R}^{n}\right): \nabla \cdot w \in L^{2}(\Omega)\right\}$. If this problem has a unique solution, we define the Dirichlet-to-Neumann map

$$
\Lambda_{\hat{\sigma}} f_{0}=\left.\nu \cdot \widehat{\sigma} \nabla v\right|_{\partial \Omega} .
$$

Our aim is to prove the following result, first announced in [GLU]: 
Theorem 3. Let $\Omega \subset \mathbf{R}^{n}, n \geq 3$, and $g=g_{i j}$ a metric on $\Omega$. Let $D \subset \Omega$ be such there is a $C^{\infty}$-diffeomorphism $F: \Omega \backslash\{y\} \rightarrow \Omega \backslash \bar{D}$ satisfying $\left.F\right|_{\partial \Omega}=I d$ and that

$$
d F(x) \geq c_{0} I, \quad \operatorname{det}(d F(x)) \geq c_{1} \operatorname{dist}_{\mathbf{R}^{n}}(x, y)^{-1}
$$

where $d F$ is the Jacobian matrix in Euclidean coordinates of $\mathbf{R}^{n}$ and $c_{0}, c_{1}>0$. Let $\widetilde{g}=F_{*} g$ and $\widehat{g}$ be an extension of $\widetilde{g}$ into $D$ such that it is positive definite in $D^{i n t}$. Finally, let $\gamma$ and $\widehat{\sigma}$ be the conductivities corresponding to $g$ and $\widehat{g}$ by (9).

Then the boundary value problem for the conductivity equation with conductivity $\widehat{\sigma}$ is uniquely solvable and

$$
\Lambda_{\hat{\sigma}}=\Lambda_{\gamma}
$$

Note that here is no diffeomorphism $H: \Omega \rightarrow \Omega$ such that $\widehat{\sigma}=H_{*} \gamma$, so the Riemannian manifolds corresponding to $\widehat{\sigma}$ and $\gamma$ cannot be the same. Also, $\widehat{\sigma}$ can be changed in arbitrary way inside $D$ without changing boundary measurements. The proof of Theorem 3 will be given below.

Example. Let $\Omega=B(0,2) \subset \mathbf{R}^{3}$ be the ball with center 0 and radius 2 . Consider $y=0 \in D=B(0,1)$ and the map $F: \Omega \backslash\{0\} \rightarrow \Omega \backslash \bar{D}$ given by

$$
F: x \mapsto\left(\frac{|x|}{2}+1\right) \frac{x}{|x|} .
$$

Let $\gamma=1$ be the homogeneous conductivity in $\Omega$ and define $\sigma=F_{*} \gamma$. Now the metric tensor $g$ and the corresponding conductivity $\sigma_{g}$ are related by $\sigma_{g}=$ $|\operatorname{det} g|^{1 / 2} g^{j k}$. Let $g$ be the metric corresponding to $\gamma$ and $\widetilde{g}$ be the metric corresponding to $\sigma$. Consider these in the standard spherical coordinates on $\Omega \backslash\{0\}$, $(r, \phi, \theta) \mapsto(r \sin \theta \cos \phi, r \sin \theta \sin \phi, r \cos \theta) \in \mathbf{R}^{3}$. With respect to these coordinates, we see that the metric $g$ and conductivity $\gamma$ correspond to the matrices

$$
g=\left(\begin{array}{ccc}
1 & 0 & 0 \\
0 & r^{2} & 0 \\
0 & 0 & r^{2} \sin ^{2} \theta
\end{array}\right), \quad \gamma=\left(\begin{array}{ccc}
r^{2} \sin \theta & 0 & 0 \\
0 & \sin \theta & 0 \\
0 & 0 & (\sin \theta)^{-1}
\end{array}\right)
$$

and $\widetilde{g}$ and $\sigma$ correspond in the annulus $1<r<2$ to the matrices

$$
\begin{aligned}
\widetilde{g} & =\left(\begin{array}{ccc}
4 & 0 & 0 \\
0 & 4(r-1)^{2} & 0 \\
0 & 0 & 4(r-1)^{2} \sin ^{2} \theta
\end{array}\right), \\
\sigma & =\left(\begin{array}{ccc}
2(r-1)^{2} \sin \theta & 0 & 0 \\
0 & 2 \sin \theta & 0 \\
0 & 0 & 2(\sin \theta)^{-1}
\end{array}\right) .
\end{aligned}
$$

Let $\widehat{\sigma}$ be a continuation of $\sigma$ that is $C^{\infty}$-smooth in $D$. Then these metrics are as in Theorem 3 and in particular $F$ satisfies (12).

To prove Theorem 3, we start with the following result: 
Proposition 1. Let $\Omega \subset \mathbf{R}^{n}, n \geq 3$, and $g=g_{i j}$ a metric on $\Omega$. Let $u$ satisfy

$$
\begin{aligned}
& \Delta_{g} u(x)=0 \quad \text { in } \Omega, \\
& \left.u\right|_{\partial \Omega}=f_{0} \in C^{\infty}(\partial \Omega) .
\end{aligned}
$$

Let $D \subset \Omega$ be such that there is a diffeomorphism $F: \Omega \backslash\{y\} \rightarrow \Omega \backslash \bar{D}$ satisfying $\left.F\right|_{\partial \Omega}=I d$. Let $\widetilde{g}=F_{*} g$ and $v$ be a function satisfying

$$
\begin{aligned}
& \Delta_{g} v(x)=0 \quad \text { in } \Omega \backslash \bar{D}, \\
& \left.u\right|_{\partial \Omega}=f_{0}, \\
& u \in L^{\infty}(\Omega \backslash \bar{D}) .
\end{aligned}
$$

Then $u$ and $F^{*} v$ coincide and have the same Cauchy data on $\partial \Omega$,

$$
\left.\partial_{\nu} u\right|_{\partial M}=\left.\partial_{\nu} F^{*} v\right|_{\partial M}
$$

where $\nu$ is unit normal vector in metric $g$ and $\widetilde{\nu}$ is unit normal vector in metric $\widetilde{g}$. Moreover, for the constant $c_{0}:=u(y)$ we have

$$
\lim _{x \rightarrow \partial D} v(x)=c_{0} .
$$

Proof. Let $g=\left(g_{i j}\right)$ be a Riemannian metric tensor defined on $\Omega \subset \mathbf{R}^{n}$.

First, we continue the $C^{\infty}$-metric $g$ to a metric $\mathfrak{g}$ in $\mathbf{R}^{n}$ such that $\mathfrak{g}_{i j}(x)=$ $g_{i j}(x)$ for $x \in \Omega$ is such that for any $y \in \mathbf{R}^{m}$ there is a positive Green's function $G(x, y)$ satisfying

$$
-\Delta_{\mathfrak{g}} G(\cdot, y)=\delta_{y} \quad \text { in } \mathbf{R}^{n}
$$

There are several easy ways to obtain this continuation. For instance, we can continue $g$ to a metric $\mathfrak{g}$ such that outside some ball $B(0, R)$ the metric is hyperbolic. This implies that the manifold $\left(\mathbf{R}^{n}, \mathfrak{g}\right)$ is non-parabolic and has a positive non-constant super-harmonic function (see $[\mathrm{Gr}]$ ). By $[\mathrm{LiT}]$ there thus exists a positive Green's function $G(x, y)$.

Next we consider the probability that Brownian motion $B_{t}^{x}$ on manifold $\left(\mathbf{R}^{n}, \mathfrak{g}\right)$ sent from the point $x$ at time $t=0$ enters an open set $U$ : Let

$$
e_{U}(x)=P\left(\left\{\text { there is } t>0 \text { such that } B_{t}^{x} \in U\right\}\right) .
$$

Let $A=\mathbf{R}^{n} \backslash U$. By Hunt's theorem (see, [H], or [Gr], Prop. 4.4) we have

$$
e_{U}(x)=s_{A}(x),
$$

where $s_{A}(x)$ is the super-harmonic potential of $A$, that is, $s_{A}(x)$ is infimum of all bounded super-harmonic functions $h$ in $\mathbf{R}^{n}$ such that $\left.h\right|_{U}=1$ and $h \geq 0$ in $\mathbf{R}^{n}$.

Let $U=B(y, r)$ and

$$
m(y, r)=\inf _{x \in \partial B(y, r)} G(x, y) .
$$

The function

$$
h_{y, r}(x)=\frac{1}{m(y, r)} \min (G(x, y), m(y, r))
$$


is positive super-harmonic function and satisfies $\left.h_{y, r}\right|_{B(y, r)}=1$. Since $e_{U}(x) \leq$ $h_{y, r}(x)$ and $\lim _{r \rightarrow 0} m(y, r)=\infty$, we see that

$$
\lim _{r \rightarrow 0} P\left(\left\{\text { there is } t>0 \text { such that } B_{t}^{x} \in B(y, r)\right\}\right)=0 .
$$

In particular, taking limit $r \rightarrow 0$ we see that the probability that $B_{t}^{x}=y$ for some $t>0$ is zero.

Now, let $\widetilde{u}$ be any solution of

$$
\begin{aligned}
& \Delta_{g} \widetilde{u}(x)=0 \quad \text { in } \Omega \backslash\{y\} \\
& \left.\widetilde{u}\right|_{\partial \Omega}=f_{0}, \\
& \widetilde{u} \in L^{\infty}(\Omega \backslash\{y\}) .
\end{aligned}
$$

Denote $f_{r}=\left.\widetilde{u}\right|_{\partial B(y, r)}$ and let $\tau(r, x) \in(0, \infty]$ be the first time when $B_{t}^{x} \in$ $\partial \Omega \cup \partial B(y, r)$. Similarly, let $\tau(0, x)$ be the hitting time to $\partial \Omega$.

Let next $\chi_{0, r}^{x}$ be a random variable defined by $\chi_{0, r}^{x}=1$ if $B_{\tau(r, x)}^{x} \in \partial \Omega$ and zero otherwise, and let $\chi_{1, r}^{x}=1-\chi_{0, r}^{x}$. Then by Kakutani's formula,

$$
\widetilde{u}(x)=\mathbb{E}\left(\chi_{0, r}^{x} f_{0}\left(B_{\tau(r, x)}^{x}\right)\right)+\mathbb{E}\left(\chi_{1, r}^{x} f_{r}\left(B_{\tau(r, x)}^{x}\right)\right) .
$$

Letting $r \rightarrow 0$ and using the fact that $\left\|f_{r}\right\|_{\infty} \leq\|\widetilde{u}\|_{\infty}$ are uniformly bounded and (15) we see $\widetilde{u}(x)=\mathbb{E}\left(f_{0}\left(B_{\tau(0, x)}^{x}\right)\right)$. Thus $\widetilde{u}(x)=u(x)$ for $x \in \Omega \backslash\{y\}$, where

$$
\begin{aligned}
& \Delta_{g} u(x)=0 \quad \text { in } \Omega, \\
& \left.u\right|_{\partial \Omega}=f_{0} .
\end{aligned}
$$

Thus we have shown that boundary value problem (16) is solvable, and that the solution is unique. Next we change this problem to an equivalent one. Let $F: \Omega \backslash\{y\} \rightarrow \Omega \backslash \bar{D}$ be a diffeomorphism that is the identity at the boundary $\partial \Omega$. Define the metric $\widetilde{g}=F_{*} g$; then the boundary value problem (17) is equivalent to

$$
\begin{aligned}
& \Delta_{g} v(x)=0 \quad \text { in } \Omega \backslash \bar{D} \\
& \left.v\right|_{\partial \Omega}=f_{0}, \\
& v \in L^{\infty}(\Omega \backslash \bar{D}) .
\end{aligned}
$$

and solutions of the problems (17) and (18) are related by $v(x)=u(F(x))$ for $x \in \Omega \backslash\{y\}$.

Clearly, the Cauchy data of the equations (17) and (18) coincide in the sense of (14). Moreover,

$$
\lim _{x \rightarrow \partial D} v(x)=c_{0}:=u(y)
$$

This concludes the proof of Prop. 1.

We remark that the application of Brownian motion above is not essential but makes the proof perhaps more intuitive. Alternatively, in the proof of Prop. 1 one can use properties of $L^{p}$-Sobolev spaces. Indeed, for $\widetilde{u}$ satisfying $\Delta_{g} \widetilde{u}(x)=0$ 
in $\Omega \backslash\{y\},\left.\widetilde{u}\right|_{\partial \Omega}=f_{0}$, and $\widetilde{u} \in L^{\infty}(\Omega \backslash\{y\})$, we can consider the extension $\widetilde{u} \in L^{\infty}(\Omega)$ that satisfies

$$
\Delta_{g} \widetilde{u}(x)=F \quad \text { in } \Omega,
$$

where $F$ is a distribution is supported in $y$. Then $F$ has to be a finite sum of derivatives of the Dirac delta distribution supported at $y$. Now, $F \in W^{-2, p}(\Omega)$ for all $1<p<\infty$, and since $\delta_{y} \notin W^{-2, p}(\Omega)$ for $p>\frac{n}{n-2}$ and the same is true for the derivatives of the delta distribution, we see that that $F=0$. This implies that $\widetilde{u}=u$.

We now turn to the proof of Theorem 3. From now on, we assume that $F: \Omega \backslash\{y\} \rightarrow \Omega \backslash \bar{D}, F(x)=\left(F^{1}(x), \ldots, F^{n}(x)\right)$ is such that condition (12) is satisfied. First, continue $\widetilde{g}$ into $D$ so that that it is positive definite in $D^{i n t}$. Next, extend $v$ inside $D$ to a function

$$
\begin{aligned}
& h(x)=v(x) \text { for } x \in \Omega \backslash \bar{D}, \\
& h(x)=c_{0} \text { for } x \in \bar{D} .
\end{aligned}
$$

Our aim is to show that $h$ is the solution of (11). Since any solution of (18) is constant on the boundary of $\partial D$ and $\widetilde{g}$ is positive definite in $D$, we see that the solution has to be constant inside $D$. Thus $h$ is the unique solution if it is a solution.

Now we are ready to show that $v$ is a solution also in the sense of distributions. First we note that when $y(x)=F^{-1}(x)$ we have $v(x)=u\left(F^{-1}(x)\right)$ and

$$
\frac{\partial v}{\partial x^{j}}(x)=\frac{\partial u}{\partial y^{k}}(y(x)) \frac{\partial y^{k}}{\partial x^{j}}(x)
$$

and since $u \in H^{1}(\Omega)$ and $\frac{\partial y^{k}}{\partial x^{j}} \in L^{\infty}(\Omega \backslash \bar{D})$ we have $v \in H^{1}(\Omega \backslash \bar{D})$. Also, as $h \in C\left(\Omega \backslash D^{i n t}\right)$ and the trace $\left.v \mapsto v\right|_{\partial D}$ is a continuous map $C\left(\Omega \backslash D^{i n t}\right) \cap$ $H^{1}(\Omega \backslash \bar{D}) \rightarrow L^{2}(\partial D)$, we see that trace $\left.v\right|_{\partial D}$ is well defined and is the constant function having value $c_{0}$. Since restrictions of $h$ to $D$ and $\Omega \backslash \bar{D}$ are in $H^{1}(D)$ and $H^{1}(\Omega \backslash \bar{D})$, respectively, and the trace from both sides of $\partial D$ coincide, we see that $h \in H^{1}(\Omega)$.

Next we show that $\sigma \nabla v \in L^{2}(\Omega \backslash \bar{D})$. Let $e_{1}, e_{2}, \ldots, e_{n}$ be the standard Euclidean coordinate vectors in $\mathbf{R}^{n}$. Then for $x \in \Omega \backslash \bar{D}$

$$
\widetilde{g}^{j k} \partial_{k} v(x)=\left(e_{j}, \nabla_{\tilde{g}} v(x)\right)_{\tilde{g}}=\left(d\left(F^{-1}\right) e_{j}, \nabla_{g} u\left(F^{-1}(x)\right)\right)_{g}
$$

and we see from (12) that this inner product is uniformly bounded. By (5) and (12), $|\operatorname{det}(\widetilde{g})(x)| \leq c_{3} \operatorname{dist}_{\mathbf{R}^{n}}\left(F^{-1}(x), y\right)^{2}$ and thus in $\Omega \backslash \bar{D}$ the functions $V^{k}(x)=|\operatorname{det} \widetilde{g}(x)|^{1 / 2} \widetilde{g}^{k i} \partial_{i} v$ satisfy

$$
\left|V^{k}(x)\right| \leq c_{4} \operatorname{dist}_{\mathbf{R}^{n}}\left(F^{-1}(x), y\right) .
$$

This implies that $\vec{V}=\left(V^{1}(x), \ldots, V^{n}(x)\right)$ is in $H(\Omega \backslash \bar{D} ; \nabla \cdot) \cap C\left(\bar{\Omega} \backslash D^{\text {int }}\right)$. Moreover, by $[\mathrm{Gr}]$ the normal trace $\left.\vec{W} \mapsto n \cdot \vec{W}\right|_{\partial D}$ is a continuous map $H(\Omega \backslash$ 
$\bar{D} ; \nabla \cdot) \cap C\left(\bar{\Omega} \backslash D^{i n t}\right) \rightarrow H^{-1 / 2}(\partial D)$, we see that $\left.n \cdot \vec{V}\right|_{\partial D}=0$. Since the normal traces of $\vec{V}$ coincide from both sides of $\partial D$ we see that $\vec{V} \in H(\Omega ; \nabla \cdot)$ and

$$
\sum_{j, k=1}^{n} \partial_{k}\left(|\operatorname{det} \widetilde{g}(x)|^{1 / 2} \widetilde{g}^{k i} \partial_{i} h\right)=\nabla \cdot \vec{V}=0 \quad \text { in } \Omega
$$

in the sense of distributions. This means that $h$ satisfies the conductivity equation in $\Omega$ in the sense of distributions. This proves Theorem 3 .

A similar approach can be used to construct a different type of counterexample. In contrast to the previous one, here the conductivity is not bounded above near the singular surface. Consider again the sets $\Omega=B(0,2)$ and $D=B(0,1)$ in $\mathbf{R}^{3}$. In spherical coordinates we define the metric $\widehat{g}$ and the conductivity $\widehat{\sigma}$ in the domain $\{1<r<2\}$ by the matrices

$$
\begin{aligned}
& \widehat{g}=\left(\begin{array}{ccc}
(r-1)^{-2} & 0 & 0 \\
0 & \rho^{2} & 0 \\
0 & 0 & \rho^{2} \sin ^{2} \theta
\end{array}\right), \\
& \widehat{\sigma}=\left(\begin{array}{ccc}
(r-1) \rho^{2} \sin \theta & 0 & 0 \\
0 & (r-1)^{-1} \sin \theta & 0 \\
0 & 0 & (r-1)^{-1} \sin ^{-1} \theta
\end{array}\right),
\end{aligned}
$$

where $\rho>0$ is a constant. Thus, $(\Omega \backslash \bar{D}, \widehat{g})$ is isometric to the product $\mathbf{R}_{+} \times S_{\rho}^{2}$ with the standard metric, where $S_{\rho}^{2}$ is a 2-sphere with radius $\rho$. Now extend the conductivity $\widehat{\sigma}$ to $\Omega$ so that it is positive definite in $D$. It can be shown that, in the domain $\Omega \backslash \bar{D}$, the equation

$$
\begin{aligned}
& \nabla \cdot \widehat{\sigma} \nabla v(x)=0 \text { in } \Omega \backslash \bar{D} \\
& \left.v\right|_{\partial \Omega}=f_{0}, \\
& v \in L^{\infty}(\Omega \backslash \bar{D})
\end{aligned}
$$

has a unique solution. Also, when $\rho$ is small enough, we can extend the definition of $v(x)$ to the whole domain $\Omega$ by defining it to have the constant value $c_{0}=\lim _{x \rightarrow \partial D} v(x)$ in $D$. The function $v(x)$ thus obtained is a solution of the boundary value problem

$$
\begin{aligned}
& \nabla \cdot \widehat{\sigma} \nabla v(x)=0 \text { in } \Omega, \\
& \left.v\right|_{\partial \Omega}=f_{0}, \\
& v \in L^{\infty}(\Omega)
\end{aligned}
$$

in the sense of distributions.

In this case, the boundary measurements do not give information about the metric inside $D$. To see this, consider $(\Omega \backslash \bar{D}, \widehat{g})$ as $\mathbf{R}_{+} \times S_{\rho}^{2}$ with coordinates $(t, \phi, \theta)$, where $t=t(r)=-\log (r-1)$, i.e., $t \in \mathbf{R}_{+}$and $\phi, \theta$ are coordinates on $S^{2}$. We see that for sets $B\left(0,1+R^{-1}\right) \subset \Omega, R>1$ in original coordinates we 
can use the superharmonic potentials

$$
h(r, \phi, \theta)=\min \left(1, \frac{\log (r-1)}{\log (R-1)}\right)=\min \left(1, \frac{t(r)}{t(R)}\right)
$$

to see that the Brownian motion sent from $x \in \Omega \backslash \bar{D}$ does not enter $D$ a.s.. By writing the solution $u$ in terms of spherical harmonics, we have

$$
u(t, \phi, \theta)=\sum_{n=1}^{\infty} \sum_{m=-n}^{n} a_{n, m} e^{-\lambda_{n} t} Y_{m}^{n}(\phi, \theta),
$$

where $\lambda_{n}=\rho^{-1} \sqrt{n(n+1)}$. Using the original coordinates $(r, \phi, \theta)$ of $\Omega \backslash \bar{D}$ we see

$$
u(r, \phi, \theta)=\sum_{n=1}^{\infty} \sum_{m=-n}^{n} a_{n, m}(r-1)^{\lambda_{n}} Y_{m}^{n}(\phi, \theta) .
$$

Thus, if $\rho$ is small enough, the solution goes to constant and its derivative to zero when $r \rightarrow 1^{+}$so fast that the solutions of the conductivity equation satisfy the equation in sense of distributions.

\section{References}

[C] A.-P. Calderón, On an inverse boundary value problem. Seminar on Numerical Analysis and its Applications to Continuum Physics (Rio de Janeiro, 1980), pp. 65-73, Soc. Brasil. Mat. Rio de Janeiro, 1980.

[GLU] A. Greenleaf, M. Lassas, and G. Uhlmann, Anisotropic conductivities that cannot be detected by EIT, Physiolog. Measure. 24 (2003), pp. 413-419.

[Gr] A. Grigoryan, Analytic and geometric background of recurrence and non-explosion of the Brownian motion on Riemannian manifolds, Bull. Amer. Math. Soc. (N.S.) 36 (1999), 135-249

[H] G. Hunt, On positive Green's functions, Proc. Nat. Acad. Sci. U. S. A. 40 (1954), 816-818.

[LU] M. Lassas, G. Uhlmann, On determining Riemannian manifold from Dirichlet-toNeumann map, Ann. Sci. École Norm. Sup. 34 (2001), 771-787.

[LTU] M. Lassas, M. Taylor, G. Uhlmann, On determining a non-compact Riemannian manifold from the boundary values of harmonic functions, Comm. Geom. Anal. to appear.

[LeU] J. Lee, G. Uhlmann, Determining anisotropic real-analytic conductivities by boundary measurements, Comm. Pure Appl. Math. 42 (1989), 1097-1112.

[LiT] P. Li, L.-F. Tam, Green's functions, harmonic functions, and volume comparison, J. Differential Geom. 41 (1995), 277-318.

[Na] A. Nachman, Global uniqueness for a two-dimensional inverse boundary value problem, Ann. of Math. (2) 143 (1996), 71-96.

[Sy] J. Sylvester, An anisotropic inverse boundary value problem, Comm. Pure Appl. Math. 43 (1990), 201-232.

University of Rochester, Rochester, NY 14618

Rolf Nevanlinna Institute, University of Helsinki, Helsinki, P.O.Box 4, Fin-00014, FinLAND

University of Washington, Seattle, WA 98195 